\title{
Metaanamorphosical Method as a Project-based Approach for the University Course on Decision Support Methods
}

\author{
Valery I. Terekhov ${ }^{1}$ and Maria A. Skvortsova ${ }^{1 *}$ \\ ${ }^{1}$ Bauman Moscow State Technical University, 2nd Baumanskaya str., 5/1, 105005, Moscow, Russia
}

\begin{abstract}
We considered project-based training as an approach to the training of engineers in modern conditions and proposed to implement it in the field of intelligent decision support methods. As a specific example, we suggested a metaanamorphization method, which allows us to activate the visual-figurative thinking mechanisms of the decision maker and facilitates decision-making in a difficult environment or finding a solution to a complex problem. We presented the mathematical formulation of the problem, a method working in real time and a description of the algorithm. The advantages of the constructed metaanamorphoses as a part of the decision support system were presented. We considered a method for calculating the integral anamorphic index combining individual anamorphic indicators of various physical nature relevant within the decision making process. Examples of constructing metaanamorphosis using the integral indicator of anamorphism are given.
\end{abstract}

\section{Introduction}

The digital revolution and related automation and robotization of production process will inevitably lead to significant changes in the labor market. Such drastic changes require new approaches to the preparation of labor resources, and, in particular, engineers. On the one hand, it is necessary to ensure the development of technological progress by the necessary engineers, and on the other hand, to give each of them the opportunity for effective work, which will be satisfying them.

To train engineers of the required level, we even now need to understand what requirements will be imposed on future engineers by potential employers, and vice versa, and how universities should work to provide training for the required number of personnel.

Federal Law "On Education in the Russian Federation" No 273-FZ of December 29, 2012 as amended in 2019 [1] says that universities are not required to product the specialists. A university provides a certain level of education, and specialist training is an additional option that can only be implemented in conjunction with the state and the business community. The way out in this situation is to change the educational process, which consists in switching from a stream-based system for obtaining knowledge to

* Corresponding author: magavrilova@bmstu.ru 
individual training programs, project-based training and the development of online education.

\section{Theoretical research}

The authors analyze the application of project-based training on a specific example - in teaching the course "Decision Support Methods", as the least resource-intensive and quickly implemented from the approaches given above.

As shown by Galan \& Rosas-Mendoza [2], the importance of project-based training is caused by the fact that it allows future engineers to choose activities according to their interests, which corresponds to their abilities. It is aimed at developing knowledge, abilities and skills in the selected subject areas.

In carrying out projects, future engineers master the algorithm of innovative creative activity, learn to independently find and analyze information, obtain and apply the knowledge gained, experiment, gain experience in solving creative problems both individually and in groups (Debnath \& Pandey, 2011, pp. 23-34).

Surveys conducted among students (Clark \& Andrews, 2017, pp. 148) on their perceptions of the usefulness of previous training sessions on four estimates (Very Useful, Useful, Neutral, Not Useful) showed the results presented in Fig. 1. It shows the types of classes by values Very Useful + Useful, in descending order. In surveys conducted among the students of Bauman Moscow State Technical University (BMSTU), the Department of Information Processing and Control Systems, the authors obtained the results shown in Fig. 2.

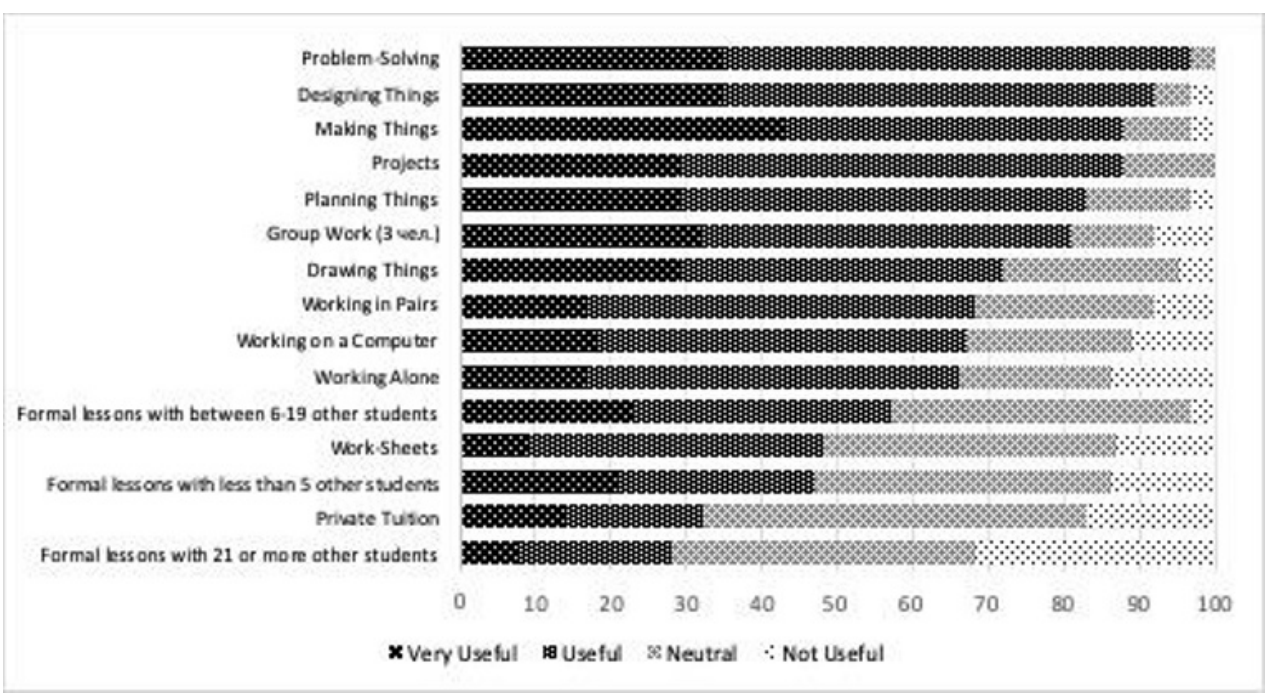

Fig. 1. Student perceptions of the usefulness of previous learning experiences (\%). 


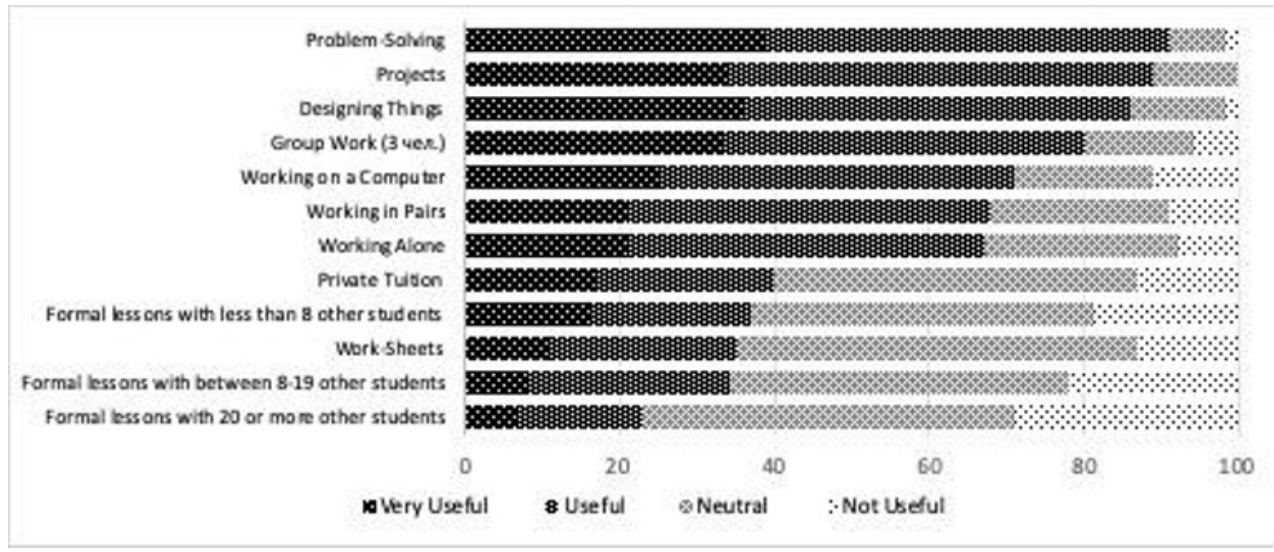

Fig. 2. Students' perceptions of the usefulness of previous training in Information Processing and Control Systems Department at Bauman Moscow State Technical University (\%).

The analysis of fig. 1 and fig. 2 shows that the students of different countries in general equally evaluate the usefulness of studies. For example, the interviewed students put the project-based approach at one of the first places, which indicates the need for its use in engineering education.

It should also be noted that one of the most important features of the project-based training method is that when it is used, the roles of participants in the project development process change significantly. The teacher ceases to be an expert and becomes a consultant and assistant, and the student acts as an active participant in the design process. At the same time, work on the project involves a mandatory assessment of what was accomplished in the course of its implementation, what were the causes of difficulties and how they can be avoided in future work.

As an example of the project-based method of training in the discipline «Decision Support Methods», we can offer a study of the decision-making process using the anamorphic method, which is one of the methods of cognitive computer graphics and can significantly facilitate decision-making, without involving complex methods data processing.

To date, various methods for actual information visualization applied for patterns identification in various subject areas exist, leading to a reduction in time for development a reasonable solution. However, for the decision-maker, a high complexity of decision problems related to input data volume growth dramatically, occurring processes dynamically increase with a simultaneous decrease in time for its production, lead to the need to use new methods for actual information visualization. Moreover, this necessity follows the law of requisite variety formulated by Ashby [3], which sets the conformity of internal diversity of the system to the diversity of its governance arrangements. This means, that a system cannot be managed using tools which are simpler than the system. Thus, for data visualization it is necessary to define a tool (method), which complexity corresponds to the complexity of the considered problem. Bellow one of these methods will be considered.

One of the ways for a fast analysis and receiving a justified solution for a wide range of applications is a required visual representation of information for the decision-maker. However, decision-making on various maps is a specific problem. For such problems information should take into account different factors and characteristics of studied process or phenomenon which can be: population density, patency of the considered area, temperature, the density of pollution and forest fires, epidemiological situation, etc. According to [4], information provided to the decision-maker in general should be visual. 
Thus, the perception and processing of this information lead to the drastic reduction of required time for analysis and, accordingly, for the adoption of a rational solution.

In addition, the accuracy and the initial data set requirements significantly depend on phase of decision making as well as on the management level at which it is produced. The higher the level of decision-maker, the more generalized and averaged information is needed to make an informed decision. Thus, there is a significant shift from a quantitative to a qualitative solution. Hence, the high-quality solution is much better and easier for visualization than quantitative, enabling a rapid decision-making maintaining its reliability [3].

The anamorphic method is proposed to use as one of the methods for initial data analysis and adoption of rational variant solutions based on actual visual information [5]. This method is related to the cognitive computer graphic which is connected to the computational intelligence methods. In this case the computer graphic is defined as a data visualization method which allows visual-figurative thinking mechanisms of decisionmakers to be activated. The method facilitates decision-making in a complex situation or finding a solution to a complex problem. The computational intelligence in the data visualization method is presented as a set of technologies, models, methods and software designed to address informal, creative tasks in various areas of human activity with the usage of logic. This set, to some extent, identifies the mental activity of man (blurred reasoning, qualitative and intuitive approach, creativity, logical conclusion, self-learning, etc.) and, in particular, decision-making, image recognition and classification [6].

It should be borne in mind that the image converted using the anamorphic method, as a rule, contains more information than before the conversion. Therefore, the initial, exclusively illustrative image is able to prompt the decision maker (DM) new thoughts and ideas for finding a solution. Thus, the original illustrative function of the image is transformed into a cognitive function. On the other hand, the cognitive function of the conversed image, made to visualize new relationships between the elements of the depicted object, turns into an illustrative function, since it begins to fulfill the function of showing an already visualized fact.

The anamorphic method is based on the concept of anamorphosis, which is defined as the transition from one visual image, built on the basis of the Euclidian metric, to another visual image. The image is based on the process or phenomenon metric using the selected index.

In the most general form, the method can be presented as follows. Fig. 3 demonstrated a part of a map coated with a square grid. Every cell of the grid corresponds to a certain parameter, for example the cross-country rate and the corresponding matrix.

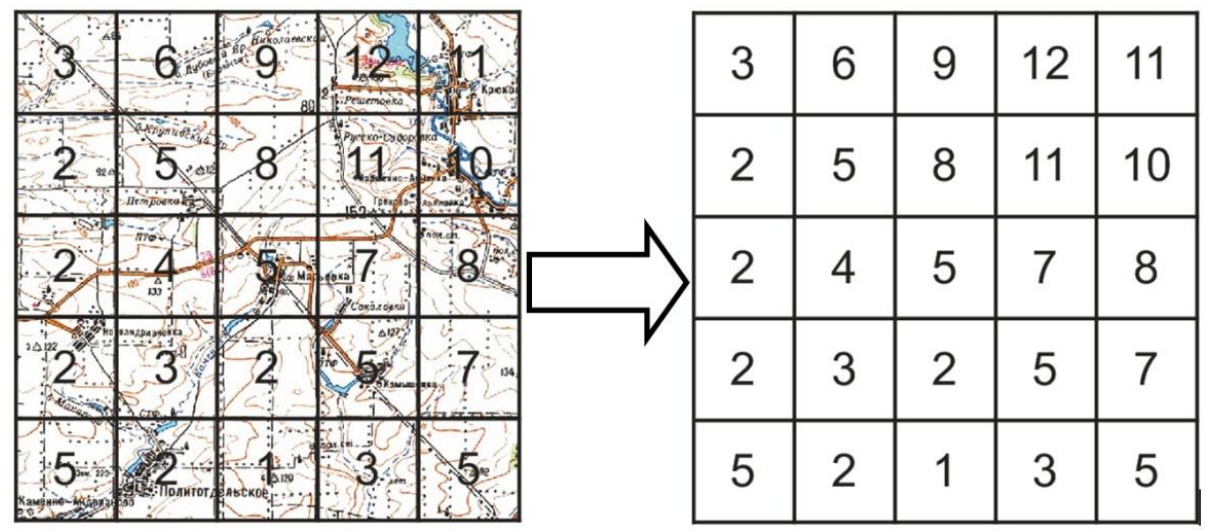

Fig. 3. The cross-country rate matrix. 
The cross-country rate matrix can be expressed as a three-dimensional matrix as shown in Fig. 4a, where the height of each bar corresponds to the value of each index in the original matrix cell. Then anamorphic operation can be presented as a superimposition of a glass on the upper side of the matrix, and pressing on it to a level that is equal to the mean value of the cross-country rate across the matrix (in our case $v_{\text {mean }}=5,52 \mathrm{~km} / \mathrm{h}$ ). As a result, the columns which were higher than the mean, reduced to this value, increasing its area - "blur", and the bars which were below the mean, "pulled up" to this level, reducing its area (Fig. 4b).

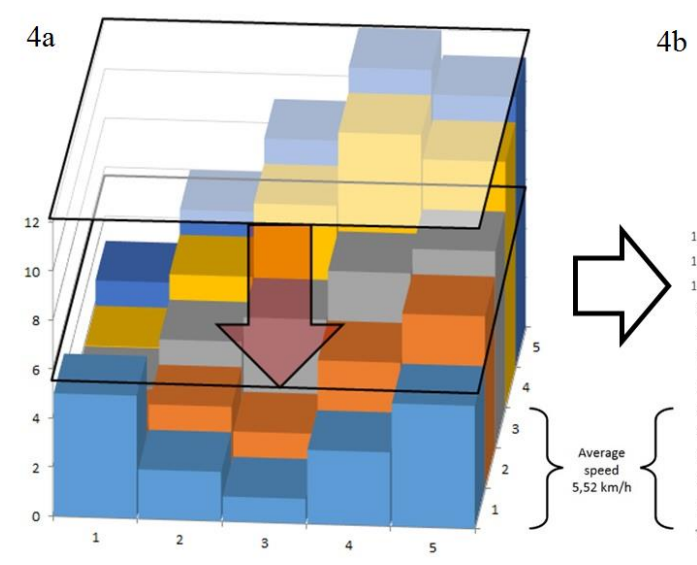

Fig. 4. Three-dimensional matrix anamorphosis of cross-country rate matrix.

Since the original matrix grid does not lose its connectivity, as a result of the anamorphic operation is a matrix shown in Fig. $4 \mathrm{~b}$ and Fig. 5.

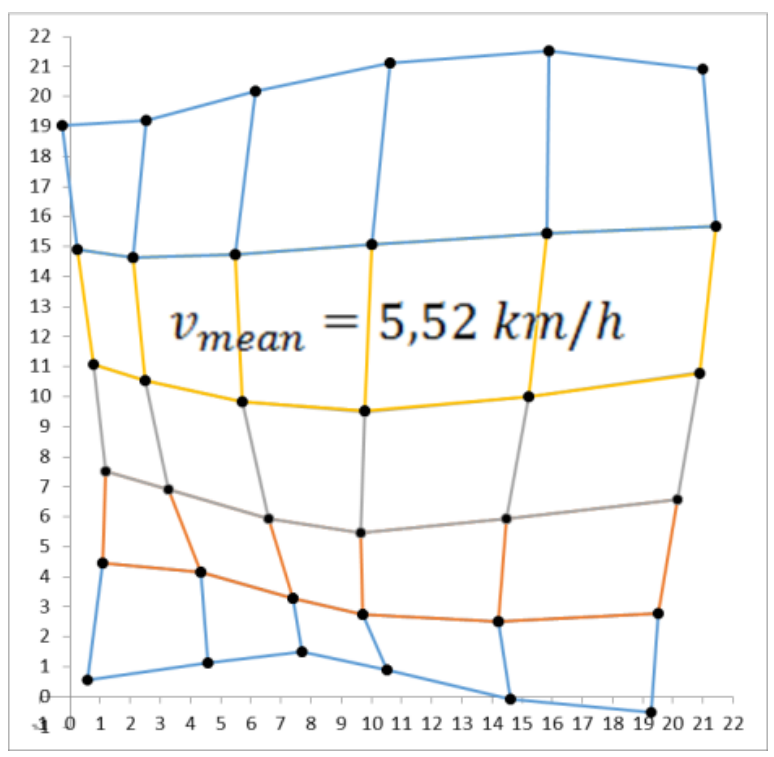

Fig. 5. Anamorphed cross-country rate matrix. 
At the same time, parameters in all cells of the resulting anamorphed matrix are identical and equal to the average value. This does possible to make a decision and carry out a visual solution modeling in the linear space of the selected parameter. It should also be noted, that the anamorphic process has reduced the solution space by unity, by averaging the indicator and taking into account its value in the form of cells.

Thus, for example, traffic routing options, on the anamorphed map in the linear space for the cross-country rate is presented in Fig. 6a, and in Euclidean space in Fig. 6b.

Inverse transformation, transferring the built anamorphosis in the linear space of the selected parameter to the original Euclidean space (Fig. 6) is anti-anamorphosis or morphing.

The current numerical methods for constructing anamorphoses [7-10], despite their diversity and ease of implementation, are very laborious for the stage of preparing the initial data, slow convergence [11-12], and violation of the integrity of the resulting visual image [13], depending on the order of the processed vertices of the cells of the matrix [12], on the order of sorting the cells and work mainly with one indicator of anamorphic [14].

Therefore, to solve the practical problems of decision making and their subsequent modeling, it is necessary to modify the anamorphic algorithm in such a way that, using existing developments, it is maximally free from the above disadvantages.

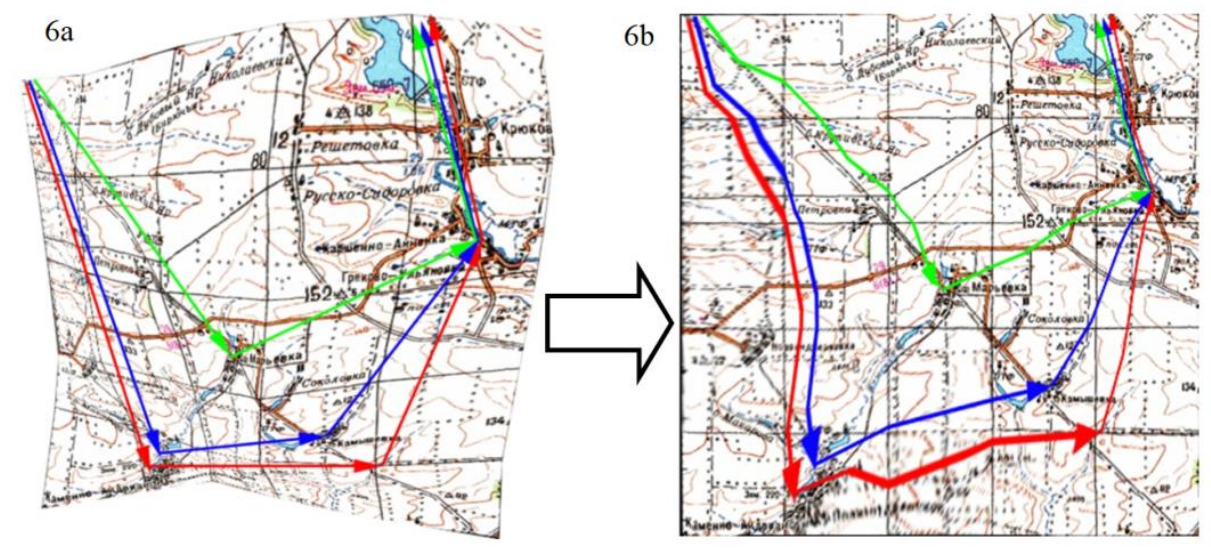

Fig. 6. Traffic routing options for transport in the linear space for the cross-country rate $-6 a$, in Euclidean space $-6 b$.

\section{Metholodgy}

The mathematical formulation of the anamorphic algorithm is considered below based on the following considerations.

$\boldsymbol{D}$ - a domain in $\boldsymbol{R}^{2}$ (areal figure, constructed based on the selected parameter), which should be anamorphed, wherein the parameter distribution is described by the function $p(z)$, defined a priori on the part of the plane $\boldsymbol{D}\left(z=(x, y)\right.$ is a point on the plain $\left.\boldsymbol{R}^{2}\right)$. Without loss of generality it can be assumed, that the function $p(z)$ density is defined on the whole plane $\boldsymbol{R}^{2}$. Thus, it can be defined as a constant outside $\boldsymbol{D}$ (the average value of $\bar{p}$ of function $p(z)$ on $\boldsymbol{D})$. Then, the transformation of anamorphosis is presented as follows.

$$
h: R^{2} \rightarrow R^{2}(h:(x, y) \mapsto(u, v))
$$

or using functions $U(x, y)$ and $V(x, y)$, where $u=U(x, y), v=V(x, y)$ are continuous, one-to-one functions. 
Transformation $h$ changes the area in the vicinity of the point $(x, y)$ with coefficient $J$.

$$
J=J(U, V)=\frac{\partial U}{\partial x} \cdot \frac{\partial V}{\partial y}-\frac{\partial U}{\partial y} \cdot \frac{\partial V}{\partial x} .
$$

The condition $p(x, y)=\bar{p}=$ const may be written as $J(U, V)=p(x, y) / \bar{p}$. Then, the problem of finding the anamorphoses is reduced to the equation (2).

$$
\frac{\partial U}{\partial x} \cdot \frac{\partial V}{\partial y}-\frac{\partial U}{\partial y} \cdot \frac{\partial V}{\partial x}=\frac{p(x, y)}{\bar{p}}
$$

where $[U(x, y), V(x, y)]$ defines the bijective transformation (Huseyn-Zade \& Tikunov 1999).

As a result of this transformation, every areal figure is deformed in accordance to a given function, which leads to a change in the general form of the entire area $\boldsymbol{D}$.

The difficulties of constructing anamorphosis using computers are as follows:

- it is necessary to control the bijectivity preservation of the transformed areal figures;

- from (2) it follows that there are infinitely many transformations satisfying this condition, i.e. the condition of a given density alignment does not define the anamorphosis clearly.

Built anamorphic image without disturbing the density constancy can be changed using any transformation that preserves the area:

1) $(u, v) \mapsto\left(k \cdot u, k^{-1} \cdot v\right)$ (stretching along one axis and contraction along another with the same coefficient);

2) $(u, v) \mapsto(u+f(v), v),(u, v) \mapsto(u, v+g(u))$ (offset horizontal and vertical lines along themselves at different distances).

In the anamorphosis construction is possible to use the conformal transformation condition (1), changing all distances by multiplying by the same coefficient which does not depend on directions (angles between the lines are preserved). The transformation which locally modifies all distances multiplication by $\sqrt{\rho(x, y)}$ is unique and does not depend on the choice of coordinate system (Huseyn-Zade \& Tikunov 1999).

It is important to note, that the conformal transformation with the linear stretch coefficient $\sqrt{\rho(x, y)}$ or with a space change coefficient equal to $\rho(x, y)$ does not always exist. This conformality condition conversion (2) may be written as follows:

$$
\left\{\begin{array}{c}
\left(\frac{\partial U}{\partial x}\right)^{2}+\left(\frac{\partial V}{\partial x}\right)^{2}=\rho(x, y) \\
\left(\frac{\partial U}{\partial y}\right)^{2}+\left(\frac{\partial V}{\partial y}\right)^{2}=\rho(x, y) . \\
\frac{\partial U}{\partial x} \cdot \frac{\partial V}{\partial y}+\frac{\partial V}{\partial x} \cdot \frac{\partial U}{\partial y}=0
\end{array}\right.
$$

This condition contains three equations with two unknown functions $U(x, y)$ and $V(x, y)$. Usually, such a system has no solutions. In order to realize such conversion, it is necessary that the density function $\sqrt{\rho(x, y)}$ satisfies the equation:

$$
\Delta \ln \rho \equiv \frac{\partial}{\partial x}\left(\frac{\partial \rho / \partial x}{\rho}\right)+\frac{\partial}{\partial y}\left(\frac{\partial \rho / \partial y}{\rho}\right)=0,
$$

where $\Delta=\frac{\partial^{2}}{\partial x^{2}}+\frac{\partial^{2}}{\partial y^{2}}$ - Laplacian.

This equation means that the curvature defined by the metric $\rho(x, y)\left(d x^{2}+d y^{2}\right)$ should be equal to 0 .

Thus, there arises a search problem among various transformations which is invariant to any change in the Cartesian coordinate system and conformal. 
Considering disadvantages of the existing anamorphic algorithms, it can be concluded that computation for algorithm realization should take into account the following points.

Firstly, it is required that at each step all partition cells affect by the shift value of points (vertices cells). Thus, it is necessary that at every step the point shift is equal to a vector shift sum resulting of the individual cells influence.

Secondly, the influence of the cell on the point should be described as displacement this point along a line, connecting it with a cell point (with its center of mass for instance). This condition is related to the algorithm invariant requirement with respect to the choice of coordinate system

Thirdly, point displacement under the influence of the cell should decrease with increasing distance from this cell.

Below is considered the method, which works in real time and takes into account aforementioned comments.

Let an areal figure has an area of $s_{i}=0,5 \cdot\left[\left(x_{i 1}-x_{i 2}\right)\left(y_{i 1}-y_{i 2}\right)+\cdots+\left(x_{i(n-1)}-\right.\right.$ $\left.\left.x_{i n}\right)\left(y_{i(n-1)}-y_{i n}\right)\right]$, where $p_{i}(x, y)=P_{i}$. In case of anamorphosis it should be deformed, thus it becomes equal to $\tilde{s}_{i}=\frac{P_{i}}{p_{i}}$, where $\bar{p}$ - mean or any other mean, which is the predetermined index value per area unit. One cell center influence to any other cell vertex is expressed as a shift this vertex in the distance:

$$
l=\left\{\begin{array}{c}
r(\tilde{R} / R-1) \text { for } r \leq R \\
\sqrt{1+\left(\tilde{R}^{2}-R^{2}\right) / r^{2}} \text { for } r>R
\end{array},\right.
$$

where $r$ - the distance between the apex of the figure and the center of affected the figure, and $\tilde{R}=\sqrt{\tilde{s}_{i} / \pi} ; R=\sqrt{s_{i} / \pi}$.

This influence describes by the radius vector of the vertex $\vec{v}$ beginning at the figure center, multiplied by the coefficient $(\tilde{R} / R-1)$ when $r \leq R$ or $\sqrt{1+\left(\tilde{R}^{2}-R^{2}\right) / r^{2}}-1$ when $r>R$. As the figure center, the gravity center can be selected.

The anamorphic algorithm can be described as follows. The vector sum of the all figures influences on vertexes and the gravity center of the considered figure is calculated at every step. Vertexes and the gravity center of the figure shift in accordance to the obtained shift vectors. For the derived configuration, new figure squares are calculated. The iteration process stops when relative deviations $N$ of all figure squares are less than the predetermined error value $\varepsilon$.

\section{Results}

The practical application of the anamorphic method is shown in the following examples.

1. Decision making to eliminate the effects of chemical pollution of the area.

The predetermined part of the area is presented as the map shown in Fig. 7, where chemical polluted zones are illustrated. 


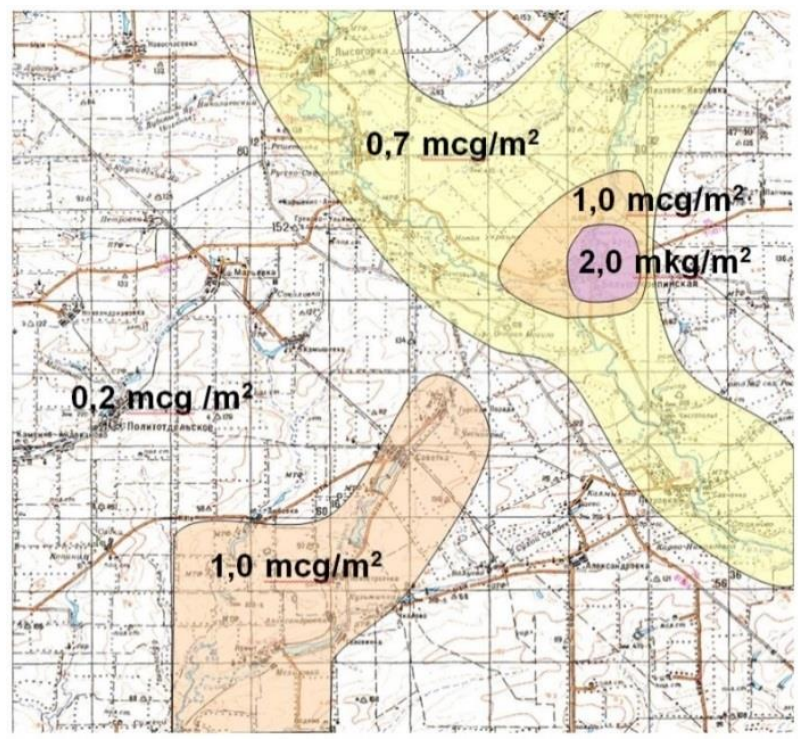

Fig. 7. A part of the map with chemical polluted areas.

The predetermined part of the area is presented as the map shown in Fig.5, where chemical polluted zones are illustrated. The part can be presented as a speed matrix for tracked vehicles (Fig.8a) and the inverse matrix (Fig.8b) of the chemical polluted areas (the maximum speed tracked vehicles - minimal chemical pollution).

Matrix partitioning into square cells is carried out in accordance with the topographic map grid.

a

\begin{tabular}{|c|c|c|c|c|c|c|c|c|c|}
\hline & & & & & & & & & \\
\hline 20, & 20,0 & 20,0 & 20,0 & 5,0 & 5,0 & 20,0 & 5,0 & 20,0 & \\
\hline 5,0 & 5,0 & 5,0 & \begin{tabular}{|l}
5,0 \\
\end{tabular} & 20,0 &, 0 & 5,0 & 5,0 & 5,0 & \\
\hline & 5,0 & 5,0 & 20,0 & \begin{tabular}{|l|l}
20,0 \\
\end{tabular} &, 0 & 5,0 & 5,0 & 5,0 & \\
\hline 20,0 & 20,0 & 20,0 & 20,0 & 5,0 & 20,0 & 20,0 & 20,0 & 20,0 & \\
\hline 5,0 & 5,0 & 5,0 & 20,0 & 5,0 & 5,0 & 5,0 & 20,0 & 5,0 & \\
\hline 5,0 & 5,0 & 5,0 & 5,0 & 5,0 & \begin{tabular}{|l}
20,0 \\
\end{tabular} & 5,0 & $5, \mathrm{C}$ & 5,0 & \\
\hline 5,0 & 5,0 & 20,0 & 20,0 & 20,0 & 20,0 & 5,0 & 20,0 & 5,0 & \\
\hline & 20,0 & 5,0 & 5,0 & 5,0 & 20,0 & 20,0 & 20,0 & 20,0 & \\
\hline & 5, & 50 & & 5,0 & 5,0 & 5 & 20, & 20,0 & \\
\hline & & & & & & & ? & 20 & \\
\hline
\end{tabular}

b

\begin{tabular}{|c|c|c|c|c|c|c|c|c|c|}
\hline & 2 & 3 & 4 & 5 & 6 & & 8 & & 10 \\
\hline 2,0 & 2,0 & 2,0 & 1,5 & 1,5 & 2,0 & 2,0 & 1,5 & 1,5 & 2,0 \\
\hline 2,0 & 2,0 & 2,0 & 1,5 & 1,5 & 1,5 & 1,5 & 1,5 & 1,5 & 2,0 \\
\hline 2,0 & 2,0 & 2,0 & 2,0 & 1,5 & 1,5 & 1,5 & 1,2 & 2,0 & 2,0 \\
\hline 2,0 & 2,0 & 2,0 & 2,0 & 2,0 & 1,5 & 1,2 & 0,2 & 2,0 & 2,0 \\
\hline 2,0 & 2,0 & 2,0 & 2,0 & 2,0 & 2,0 & 1,5 & 1,5 & 2,0 & 2,0 \\
\hline 2,0 & 2,0 & 2,0 & 2,0 & 2,0 & 1,2 & 2,0 & 1,5 & 1,5 & 2,0 \\
\hline 2,0 & 2,0 & 2,0 & 2,0 & 1,2 & 1,2 & 2,0 & 2,0 & 1,5 & 1,5 \\
\hline 2,0 & 2,0 & 1,2 & 1,2 & 1,2 & 2,0 & 2,0 & 2,0 & 2,0 & 1,5 \\
\hline 2,0 & 2,0 & 1,2 & 1,2 & 1,2 & 2,0 & 2,0 & 2,0 & 2,0 & 2,0 \\
\hline 2,0 & 2,0 & 1,2 & 1,2 & 2,0 & 2,0 & 2,0 & 2,0 & 2,0 & 2,0 \\
\hline
\end{tabular}

Fig. 8. Zone matrix: a - tracked vehicle traveling speeds $(\mathrm{km} / \mathrm{h}) ; \mathrm{b}$ - chemical polluted areas $\left(\mathrm{mkg} / \mathrm{m}^{2}\right)$.

Since the algorithm of the method represents every cell as a circle with square $\tilde{s}_{i}=P_{i} / \bar{p}$, then it is possible to use the integral indicator matrix, taking into account chemical polluted areas and tracked vehicle traveling speeds by averaging the circular areas of the corresponding matrix cells

In such a matrix, the integral area of each cell will be determined as:

$$
\tilde{s}_{i \text { int }}-\left(\tilde{s}_{i 1}+\tilde{s}_{i 2}+\ldots+\tilde{s}_{i R}\right) / R,
$$

where $R$ - number of indicators considered.

In example 1, such indicators will be indicators of chemical pollution of the area and the speed of movement of tracked vehicles. In other words, it is possible to use various parameters for anamorphosis for different physical nature.

Taking into account that after the integrated matrix anomorfosis process, its cells have 
changed the geometry and the integral indicator of the anomorfosis for every cell has been equalized to the average across the matrix (Fig. 9). This is possible to use on this matrix a uniform linear space of the integral parameter. For instance, in the example the shortest distance between any objects is a straight line, taking into account tracked vehicle traveling speeds and chemical pollutions. It is also possible to conduct the reverse conversion - antianamorphosis (morphing), which transfers the built anamorphosis to the original Euclidean space.
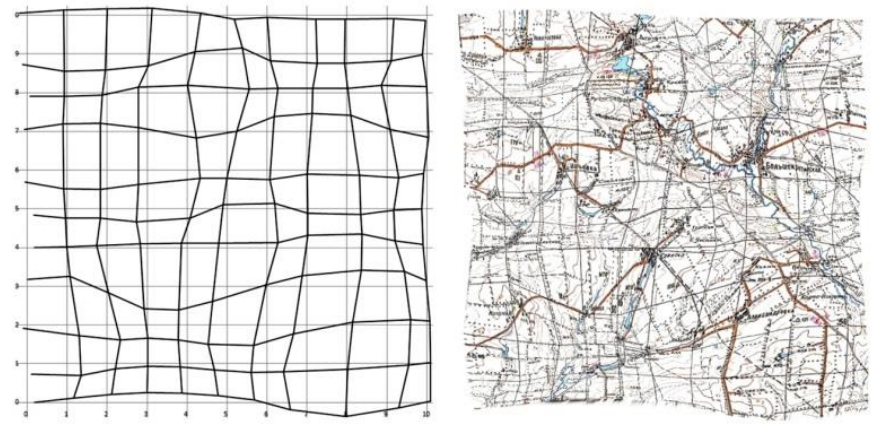

Fig. 9. Integral matrix anamorphosis and the corresponding map of the area which is built using morphing.

Fig. 10 demonstrates an example of a decision for liquidation the chemical pollution consequences considering tracked vehicle traveling speeds and chemical pollutions.
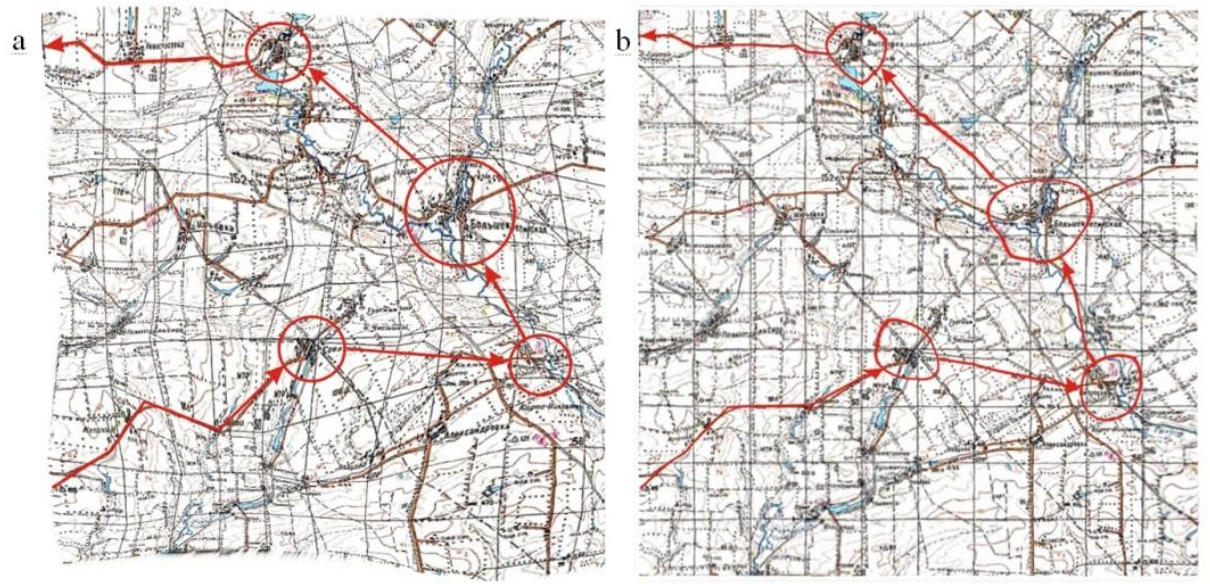

Fig. 10. Decision for liquidation the chemical pollution consequences: $a-$ on the anamorphed map; $b$ - anamorphed map morphing into Euclidean space.

2. Decision-making on determining the allocated resources to the population of the North Caucasian Federal District and the Astrakhan Region of the Russian Federation, considering the average population growth and per capita income of 2015-2016 [16-20].

In this example, the integral anamorphic indicator should be calculated with account of the average population growth and per capita income in 2015-2016 based on the values of these indicators for each Federal subject and the Astrakhan region. The results of this calculation are presented in Fig.11. 

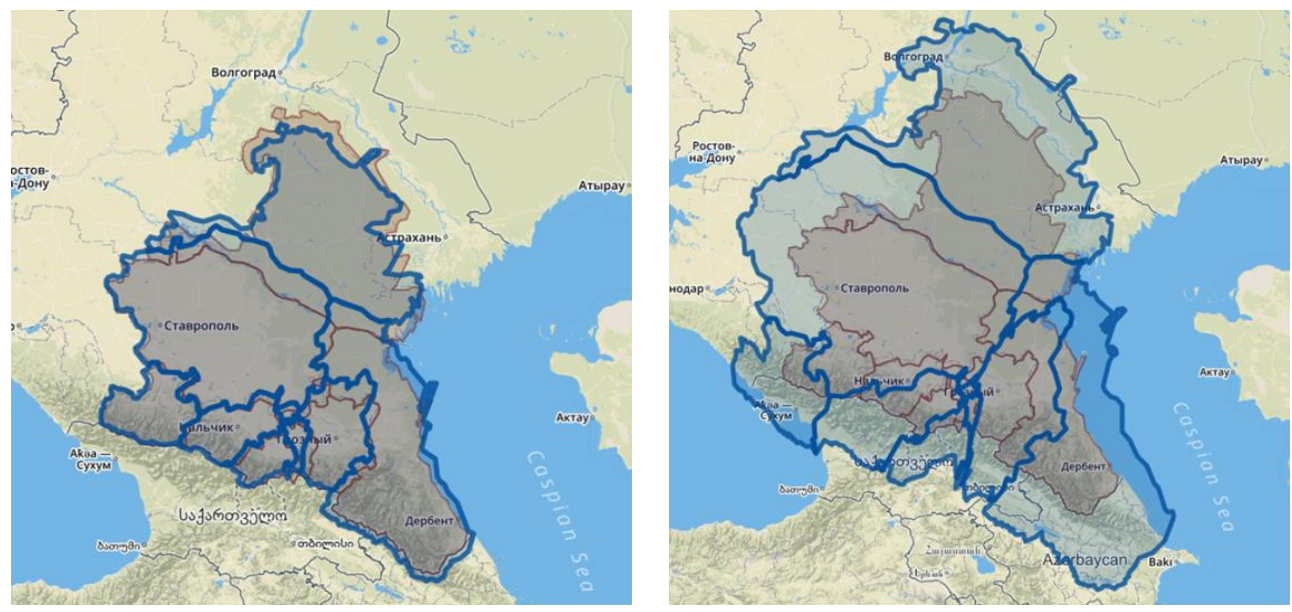

Fig. 11. Anamorphosis of per capita income and anamorphosis of the integral indicator (per capita income and population growth) in 2015-16.

It should be noted that the calculation of the anamorphosis presented in Fig. 11 was made based on coordinates describing the boundaries of objects, and not based on the digitization of these objects by square matrices (Fig. 9). At the same time, the value of the indicator of income and population growth is attributed to the corresponding object, and its administrative center is appointed as the center of it.

Anamorphosis Analysis in Fig. 11 shows that the main efforts to allocate resources to the population of the North Caucasus Federal District and the Astrakhan Region should be concentrated on the Stavropol Territory and the Chechen Republic, which gave the largest increase in their area, taking into account the integral indicator of per capita income and population growth in 2015-16.

\section{Conclusion}

Thus, the anamorphic method, which serves to visually present to the decision maker the relevant information, can be used as a project-based method of training in the discipline "Decision Support Methods" in a group of three students (Fig. 1, Fig. 2) for solutions primarily of practical problems associated with the terrain and comprehensively considering its various properties and characteristics.

As a result of the research, we can conclude that the advantages of the anamorphic method, when used in the design method of training, are:

- Decision making in a uniform space of the selected parameter;

- The ability to compute anamorphosis taking into account the integral anamorphic parameter (for example population density, tracked vehicle traveling speeds, the pollution concentration in the area);

- Reduction the decision making dimension on parameter numbers used by anamorphing the parameters, since this parameters are recomputed into the forms of considered objects;

- Visibility - a combination of the cells size and their possible coloring by given parameter ranges (Fig.3 - traveling speeds, which allows to identify possible directions, lanes and routes for efficient movements.);

- Initial speed matrix anamorphosis, both for the average travelling speed and for any predetermined speed, allow possible directions and movement areas of these rates to be identified; 
- The ability to design and to analyze action scenarios based on the recomputed anamorphosis. This anamorphosis takes into account tasks related to dynamic changes in cross-country areas (or a value taken as an anamorphosis parameter) and the adoption of relevant decisions.

- The ability to calculate anamorphoses taking into account the complex anamorphic parameter (e.g. population density, tracked vehicle traveling speeds, the pollution concentration in the area).

\section{References}

1. Federal Law, On Education in the Russian Federation, No. 273-FZ of December 29, 2012 as amended in 2019 (2012). http://zakon-ob-obrazovanii.ru/

2. I.O. Galan, A.M. Rosas-Mendoza, A Project-Based Learning Approach: Developing Mathematical Competences in Engineering Students, Handbook of Research on Driving STEM Learning With Educational Technologies, IGI Global, pp. 107-128 (2017)

3. A.D. Ursul, Information: methodological aspects, The science (1971)

4. V.D. Glezer \& I. I. Zuckerman, Information and vision, Publishing House of the Academy of Sciences of the USSR [Leningrad Branch] (1961)

5. V.I. Terekhov, The use of cognitive computer graphics to visualize relevant information to decision makers, Bulletin of BMSTU Ser.: Instrumentation, p. 109 (2012)

6. Yu. Zaichenko \& M. Zgurovsky, System analysis of directions of computational intelligence, Information content \& processing, p. 220 (2014)

7. S.M. Huseyn-Zade \& V.S. Tikunov (1999). Anamorphosis: what is it. M .: Editorial UrSS, 59-63.

8. D. Dorling, Area cartograms: their use and creation, Concepts and techniques in modern geography (1996)

9. M. Alam, S. G. Kobourov \& S. Veeramoni, Quantitative measures for cartogram generation techniques, Computer Graphics Forum, June 2015, Vol. 34, No. 3, pp. 351360 (2015)

10. S. Nusrat, M. J. Alam \& S. Kobourov, Evaluating cartogram effectiveness, IEEE Transactions on Visualization and Computer Graphics (2016)

11. W. Tobler, Cartograms and cartosplines, Proceedings of the 1976 Workshop on Automated Cartography and Epidemiology, pp. 53-58 (1976)

12. P.V. Petrov, S.N. Serbenyuk \& V.S. Tikunov, An analytical way to create anamorphic cartographic images, Bulletin of Moscow University, Ser, 5, p. 56 (1983)

13. S. Selvin, D. Merrill, J. Schulman, S. Sacks, L. Bedell \& L. Wong, Transformations of maps to investigate clusters of disease, Social Science \& Medicine, 26(2), pp. 215-221 (1988)

14. J.A. Dougenik, N.R. Chrisman \& D.R. Niemeyer, An Algoritm To Construct Continuous Area Cartograms* (1985)

15. M. Skvortsova and V. Terekhov, Study of The Possibility of Applying Intellectual Methods in Creating Risk Analysis Decision Support System in The Socio-Economic Sphere, 2019 IEEE Conference of Russian Young Researchers in Electrical and Electronic Engineering (EIConRus), Moscow, Russia, pp. 1892-1896 (2019) 
16. M. Skvortsova, et al, Hybrid Intelligent System for Risk Assessment based on Unstructured Data, Proceedings of the 2017 IEEE Russia Section Young Researchers in Electrical and Electronic Engineering Conference, pp. 560-564 (2017). DOI: 10.1109/EIConRus.2017.7910616.

17. V. Chernenkiy, et al., The hybrid intelligent information system approach as the basis for cognitive architecture, Procedia computer science, Sci. 145, pp. 143-152 (2018)

18. V. Terekhov, I. Chernenky, S. Buklin, A. Yakubov, Cognitive Visualization in Management Decision Support Problems, Optical Memory and Neural Networks, Vol. 28, No. 1, pp. 27-35 (2019)

19. A.V. Proletarsky, D.V. Berezkin, Yu.E. Gapanyuk, I.A. Kozlov, I.A. Popov, R.S. Samarev, V. I. Terekhov, Methods of situation analysis and graphical visualization of big data streams, Herald of the Bauman Moscow State Technical University, [Series Instrument Engineering], No. 2 (119), pp. 98 - 123 (2018) 